\title{
RESIDUOS SÓLIDOS URBANOS E INDUSTRIALES: SITUACIÓN Y GESTIÓN EN ESPAÑA
}

\author{
POR
}

\author{
ALFONSO MULERO MENDIGORRI
}

Producción, composición y tratamiento de los residuos en España

En la actualidad los residuos urbanos e industriales han alcanzado en España la categoría de gran problema medioambiental, o al menos esto es lo que se deduce tras considerar su volumen total, su variedad cualitativa, su extendida presencia territorial y sus previsiones de crecimiento. Esta aseveración se refuerza si tenemos en cuenta los escasos avances aplicados a la gestión de residuos hasta hace pocos años y el considerable porcentaje de vertidos incontrolados que hoy se detecta en nuestro país.

La gran variedad implícita en el concepto de «residuo» obliga, cuando menos, a abordar su estudio partiendo de las dos categorías básicas existentes: los residuos sólidos urbanos (R.S.U.) y los residuos industriales (R.I.). Los primeros engloban de forma genérica a los producidos por cualquier actividad en los núcleos de población o sus zonas de influencia. Esto significa que los residuos sólidos urbanos son algo más que los desechos generados en el ámbito doméstico, ya que se ha de contemplar el conjunto de otras actividades generadoras de residuos ubicadas en el medio urbano (mercados, construcción, hospitales, etc.)

Alfonso Mulero Mendigorri. Depto. de Geografía y Ciencias del Territorio. Universidad de Córdoba.

Estudios Geográficos Tomo LIX, n. ${ }^{\circ} 232$, julio-septiembre 
(Otero del Peral, 1988); por otra parte, los provenientes de diversos procesos industriales conforman una categoría diferenciada en virtud de su especificidad y su mayor poder contaminante, lo que en parte viene determinado porque una porción considerable de ellos es catalogada como tóxica y peligrosa.

También es heterogénea la responsabilidad en la gestión de los residuos en nuestro país. El papel del Gobierno central —representado hasta 1996 por el Ministerio de Obras Públicas Transporte y Medio Ambiente (MOPTMA) y desde entonces por el Ministerio de Medio Ambiente (MMA) - está circunscrito a la financiación, la coordinación, la investigación y la redacción de planes nacionales; las Comunidades Autónomas tienen a su cargo la elaboración de planes regionales de residuos sólidos urbanos, y la ejecución de la normativa nacional (sobre residuos peligrosos, recuperación de suelos contaminados, etc.); los municipios llevan a cabo la gestión de los residuos sólidos urbanos generados en sus áreas y, finalmente, las empresas industriales son responsables del adecuado tratamiento de los residuos peligrosos que producen.

De entrada las cifras globales permiten adivinar la magnitud del problema y sus consecuencias: Las últimas estimaciones oficiales disponibles ${ }^{1}$ indican que en España se han generado 14,9 millones de toneladas de residuos sólidos urbanos (1995) —un 50\% más que en 1985y 13,3 millones de toneladas de residuos industriales (1994), de los cuales 3,4 millones han sido tóxicos y peligrosos. El vertiginoso aumento de los mismos ha sido una de las inevitables consecuencias del devenir reciente de la sociedad y economía españolas; éste ha comportado diversos procesos que han contribuido a situar a los residuos en la cúspide de las preocupaciones medioambientales del estado español; recordemos, entre otros, el incremento demográfico, la fuerte concentración de la población en núcleos urbanos, el aumento del nivel de vida y del consumo per capita, los procesos industriales agresivos con el entorno, la actuación política tardía y escasamente preventiva, la escasa formación medioambiental del español, el uso intensivo de embalajes y envases sin retorno, etc.

${ }^{1} \mathrm{El}$ artículo ha sido revisado en el mes de abril de 1998 y las últimas cifras oficiales disponibles siguen estando referidas al año 1995, tal y como se aclara en el volumen sobre el Medio Ambiente en España 1996 (Madrid, Ministerio de Medio Ambiente, 1997) de reciente publicación. 
Los residuos sólidos urbanos.-Conviene aclarar desde el principio que la mayor parte de los ayuntamientos españoles han centrado su atención en el campo específico de los residuos domésticos y sólo en los últimos años se va generalizando en los entes locales un servicio de recogida de enseres, electrodomésticos, escombros, y todos aquéllos que se integran también en la categoría de R.S.U. Según las estimaciones oficiales, las materias de este tipo recogidas suponen sólo el $5 \%$ (unas $700.000 \mathrm{Tn}$.) respecto del total de residuos domésticos contabilizados en la actualidad; sin embargo, todavía escapan a la contabilidad oficial la mayor parte de R.S.U. no domésticos, y se desconoce con exactitud la cuantía de su volumen y su destino final.

No obstante, una vez señalado este extremo y aclarada la frecuente confusión entre R.S.U. y residuos domésticos ${ }^{2}$, podemos afirmar que hoy su problemática se manifiesta al menos de tres formas:

$1^{\circ} \quad$ La producción de residuos ha crecido vertiginosamente en los últimos años y no se espera un cambio de signo en esta tendencia.

$2 .^{\circ} \quad$ La diversidad de las materias residuales dificulta su gestión y exige a las administraciones el diseño de estrategias muy variadas para su control y recogida.

$3 .^{\circ} \quad$ Existen distintos sistemas de eliminación y tratamiento, pero su eficacia viene siendo cuestionada, al menos parcialmente. No se olvide que todavía 2,6 millones de toneladas (18\%) se depositan en vertederos incontrolados.

Efectivamente, la generación de basuras ha experimentado un notable incremento en nuestro país, lo que, en parte, es consecuencia de la evolución del nivel de vida y de las pautas de consumo. A este respecto lo verdaderamente preocupante es que esta tendencia parece bien afianzada y no ofrece signos de cambio a medio plazo (cuadro I). Lo dicho no es, por supuesto, privativo de España, ya que en el conjunto de la Unión Europea la producción de R.S.U. ha crecido más del $45 \%$ en las dos últimas décadas, una evolución muy significativa si la contras-

\footnotetext{
${ }^{2}$ Estos conceptos, a pesar de su diferente significado, se vienen empleando indistintamente en las publicaciones sobre residuos, habiéndose generalizado el uso de R.S.U. como acepción general y comúnmente aceptada, motivo por el cual la emplearemos a partir de ahora.
} 
CUADRO I

EVOLUCIÓN DE LA PRODUCCIÓN Y DEL TRATAMIENTO DE R.S.U. EN ESPAÑA

\begin{tabular}{l|c|c|c|c|c|c|c|c|c}
\hline Año & $\begin{array}{c}\text { Vertido } \\
\text { incontro- } \\
\text { lado (tn.) }\end{array}$ & $\begin{array}{c}\text { Porcen- } \\
\text { taje }\end{array}$ & $\begin{array}{c}\text { Vertido } \\
\text { contro- } \\
\text { lado (tn.) }\end{array}$ & $\begin{array}{c}\text { Porcen- } \\
\text { taje }\end{array}$ & $\begin{array}{c}\text { Compos- } \\
\text { taje } \\
\text { (tn.) }\end{array}$ & $\begin{array}{c}\text { Porcen- } \\
\text { taje }\end{array}$ & $\begin{array}{c}\text { Incine- } \\
\text { ración } \\
\text { (tn.) }\end{array}$ & $\begin{array}{c}\text { Porcen- } \\
\text { taje }\end{array}$ & $\begin{array}{c}\text { Total } \\
\text { (tn.) }\end{array}$ \\
\hline 1978 & 4.927 .047 & 61,3 & 1.812 .000 & 22,6 & 778.700 & 9,7 & 510.000 & 6,4 & 8.027 .747 \\
1986 & 3.203 .735 & 30,3 & 4.779 .725 & 45,2 & 2.042 .400 & 19,3 & 541.840 & 5,2 & 10.567 .700 \\
1990 & 3.309 .347 & 26,4 & 6.066 .182 & 48,4 & 2.563 .720 & 20,4 & 606.395 & 4,8 & 12.545 .952 \\
1992 & 4.656 .914 & 33,6 & 7.053 .818 & 51,1 & 1.467 .525 & 10,6 & 649.398 & 4,7 & 13.827 .655 \\
1994 & 3.538 .935 & 24,8 & 8.362 .284 & 58,5 & 1.770 .061 & 12,3 & 625.022 & 4,4 & 14.296 .302 \\
1995 & 2.628 .042 & 17,6 & 9.506 .354 & 63,7 & 2.086 .347 & 13,9 & 693.491 & 4,7 & 14.914 .235 \\
\hline
\end{tabular}

Fuente: Elaboración propia a partir de datos básicos de M.O.P.U., M.O.P.T., M.O.P.T.M.A y M.M.A.

CUADRO II

LOS R.S.U. EN LAS COMUNIDADES AUTÓNOMAS, SEGÚN ORDEN DE PRODUCCIÓN TOTAL

\begin{tabular}{l|r|r|c}
\hline & Toneladas/año & Porcentaje & Kg/habitante/año \\
\hline Cataluña & 2.700 .945 & 18,11 & 442 \\
Madrid & 2.374 .335 & 15,92 & 472 \\
Andalucía & 2.202 .343 & 14,77 & 313 \\
Valencia & 1.260 .338 & 8,45 & 321 \\
Castilla-León & 1.029 .036 & 6,90 & 401 \\
Galicia & 814.229 & 5,46 & 299 \\
Canarias & 759.129 & 5,09 & 464 \\
País Vasco & 692.809 & 4,64 & 328 \\
Castilla-La Mancha & 590.861 & 3,96 & 358 \\
Baleares & 413.850 & 2,77 & 555 \\
Extremadura & 412.631 & 2,89 & 391 \\
Murcia & 394.494 & 2,65 & 372 \\
Asturias & 381.258 & 2,56 & 347 \\
Aragón & 356.691 & 2,39 & 292 \\
Navarra & 210.211 & 1,41 & 401 \\
Cantabria & 183.875 & 1,23 & 347 \\
La Rioja & 88.019 & 0,59 & 328 \\
Melilla & 25.052 & 0,17 & 393 \\
Ceuta & 24.129 & 0,16 & 330 \\
$\quad$ ESPAÑA & 14.914 .235 & 100 & 378 \\
\hline
\end{tabular}

Fuente: Medio Ambiente en España, 1996. (Ministerio de Medio Ambiente, 1997). Elaboración propia. 
tamos con el débil aumento demográfico de este espacio geográfico (Arce, R. et al., 1996). Sin embargo, siendo este fenómeno una constante en los ámbitos desarrollados, no puede ignorarse que existen notables diferencias regionales que demuestran la estrecha relación existente entre la generación de basuras y el entorno socioeconómico del habitante urbano. El cuadro II puede servir para aproximarnos a esta cuestión, y lo primero y más importante es que sólo tres autonomías producen casi la mitad (48,8 \%) de los residuos domésticos españoles: Cataluña, Madrid y Andalucía. En principio esta concentración suele asociarse exclusivamente con el volumen demográfico de este trío regional, por lo que a veces se olvida que esta relación no es directamente proporcional y que existen otros ingredientes diferenciadores. Por ello, mientras que la media anual española se sitúa en 378 kilogramos de basura por habitante, determinadas Comunidades superan ampliamente esta cifra, destacando Baleares (555), Madrid (472), Canarias (464), Cataluña (442), Castilla-León (401) y Navarra (401). En consecuencia a la hora de abordar este problema habrán de tenerse en cuenta diversos factores cuya incidencia es desigual de unos a otros ámbitos: nivel de renta, importancia del turismo - clave en los casos extremos de Baleares y Canarias-, pautas de consumo, existencia de campañas de sensibilización y educación ambiental, percepción que los propios habitantes tienen del problema, etc. La renta per cápita, siendo un elemento clave, no es el único válido a la hora de explicar los contrastes regionales en producción de basuras, como así lo demuestran situaciones tan dispares como las de Extremadura - baja renta per cápita y alta generación de residuos - o Valencia — alta renta y baja producción-.

Otro atributo que caracteriza a los R.S.U. es la heterogeneidad de su composición, lo que dificulta su control y manejo. En España la materia orgánica encabeza la lista de sustancias residuales con un $44 \%$ del total, seguida por el papel (21\%), el plástico (11\%) y el vidrio (7\%). El $17 \%$ restante se compone de cantidades inferiores de materias muy diversas como metales, textiles, gomas, maderas, etc. Es cierto que el peso de estos ingredientes, a medida que descendemos de escala, varía considerablemente según el entorno rural o urbano en que nos encontremos, su nivel de riqueza e incluso la época del año. Sin embargo, a escala estatal las diferencias entre los países desarrollados son mínimas y la composición expresada anteriormente se man- 
tiene, con puntuales excepciones ${ }^{3}$, para el conjunto de la Unión Europea.

Entre otros factores, la diversidad de materias que integran las basuras urbanas españolas convierte en necesaria la existencia de varios sistemas para su eliminación y tratamiento. Como aproximación a la situación de España es suficiente con observar el cuadro III, a partir del cual se aprecian las dos grandes cuestiones en las que nos interesa incidir. La primera y más preocupante consiste en que aún en 1995 casi el $18 \%$ (más de 2,6 millones de toneladas) de los residuos domésticos tienen como destino final alguno de los cientos de vertederos incontrolados existentes en territorio español; lo que no se contradice con que, en términos relativos, podamos hablar de tendencia muy positiva desde el momento en que sólo tres años atrás este parámetro ascendía al $34 \%$. El segundo aspecto clave se deriva de la abrumadora diferencia en el grado de utilización de los distintos sistemas que se aplican al 82\% restante de residuos: vertederos controlados, compostaje e incineración. Así, resulta abrumador el peso del vertido controlado $(63,7 \%)$ y el rechazo a los sistemas de incineración (4,6 \%). Estos últimos, paradójicamente, gozan de una importancia notable en diversos países de nuestro entorno; la aplicación media de la incineración en los países de la Unión Europea se sitúa en torno al $22 \%$-donde parte del residuo se considera un combustible o recurso energético más-, pero este porcentaje se eleva notablemente en países como Francia (37 \%) y Holanda (34 \%). El rechazo español es aún más claro si observamos las opciones de tratamiento adoptadas en las diversas comunidades, porque muestran con claridad que el citado $4,6 \%$ se deriva mayoritariamente de la destacada aplicación del sistema en Cataluña, pues la mayoría de las regiones no lo emplean aún, y otras que ya lo hacen - como Canarias, Cantabria, Navarra y País Vasco- sólo queman una porción mínima de sus basuras. Y algo similar ocurre con el empleo de las técnicas de compostaje, sólo desarrolladas con importancia notable en Valencia, Madrid y Murcia, si la relacionamos con su volumen total de desechos.

${ }^{3}$ Una de estas excepciones es la que ejemplifican Holanda y el Reino Unido, con un $52 \%$ y un $19 \%$ respectivamente de materia orgánica en la composición de sus residuos, según recogen Arce, R. et al. (1996). 


\section{CuAdro III}

SITUACIÓN DE LA ELIMINACIÓN Y EL TRATAMIENTO DE R.S.U. EN LAS COMUNIDADES AUTÓNOMAS ESPAÑOLAS (TONELADAS/AÑO)

\begin{tabular}{l|r|r|c|c|c|c|c|c}
\hline & $\begin{array}{c}\text { Vertido } \\
\text { incon- } \\
\text { trolado }\end{array}$ & $\begin{array}{c}\text { Porcen- } \\
\text { taje }\end{array}$ & $\begin{array}{c}\text { Vertido } \\
\text { contro- } \\
\text { lado }\end{array}$ & $\begin{array}{c}\text { Porcen- } \\
\text { taje }\end{array}$ & $\begin{array}{c}\text { Incine- } \\
\text { ración }\end{array}$ & $\begin{array}{c}\text { Porcen- } \\
\text { taje }\end{array}$ & $\begin{array}{c}\text { Compos- } \\
\text { taje }\end{array}$ & $\begin{array}{c}\text { Porcen- } \\
\text { taje }\end{array}$ \\
\hline Andalucía & 418.883 & 15,9 & 1.624 .929 & 17,1 & - & - & 158.531 & 7,6 \\
Aragón & 37.089 & 1,4 & 319.602 & 3,4 & - & - & - & - \\
Asturias & 3.132 & 0,1 & 378.126 & 4,0 & - & - & - & - \\
Baleares & 126.011 & 4,8 & 247.839 & 2,6 & - & - & 40.000 & 1,9 \\
Canarias & 75.563 & 2,9 & 619.430 & 6,5 & 15.126 & 2,2 & 49.004 & 2,3 \\
Cantabria & 10.334 & 0,4 & 167.652 & 1,8 & 5.899 & 0,8 & - & - \\
Castilla-La Mancha & 348.189 & 13,2 & 201.672 & 2,1 & - & - & 41.000 & 2,0 \\
Castilla y León & 314.443 & 11,9 & 741.593 & 7,8 & - & - & - & - \\
Cataluña & 16.200 & 0,6 & 1.890 .582 & 19,9 & 637.640 & 92,0 & 156.823 & 7,5 \\
Extremadura & 230.631 & 8,8 & 182.000 & 1,9 & - & - & - & - \\
Galicia & 542.301 & 20,6 & 271.978 & 2,9 & - & - & - & - \\
Madrid & 7.331 & 0,3 & 1.777 .258 & 18,7 & - & - & 589.746 & 28,3 \\
Murcia & 161.222 & 6,1 & - & - & - & - & 233.272 & 11,2 \\
Navarra & 29.603 & 1,1 & 160.623 & 1,7 & 3.714 & 0,5 & 16.271 & 0,8 \\
País Vasco & 98.337 & 3,7 & 563.060 & 5,9 & 31.412 & 4,5 & - & - \\
La Rioja & 4.519 & 0,2 & 83.500 & 0,9 & - & - & - & - \\
Valencia & 175.078 & 6,7 & 283.560 & 3,0 & - & - & 801.700 & 38,4 \\
Ceuta & 24.129 & 0,9 & - & - & - & - & - & - \\
Melilla & 5.052 & 0,2 & 20.000 & 0,2 & - & - & - & - \\
\cline { 2 - 8 } TOTAL & 2.628 .043 & 100 & 9.506 .354 & 100 & 693.491 & 100 & 2.086 .347 & 100 \\
\hline
\end{tabular}

Fuente: Medio Ambiente en España, 1996. (Ministerio de Medio Ambiente, 1997). Elaboración propia

Los residuos industriales: El problema de los residuos peligrosos.La magnitud del problema que plantean este tipo de desechos puede considerarse de menor alcance cuantitativo, debido a la inferior cantidad de los mismos y a su localización menos generalizada, pero desde un punto de vista cualitativo - según la degradación ambiental que provocan- sus consecuencias son aún mayores que en el caso anterior. Como apuntábamos arriba, las cifras recientes ofrecidas por la Secretaría de Estado de Medio Ambiente indican que la industria española origina en la actualidad 13,3 millones de toneladas de residuos, de los que preocupan especialmente los 3,4 millones de toneladas clasificadas como peligrosas (cuadro IV), ya que el resto, por su composición y carácter inerte, es considerado asimilable a los residuos sólidos urbanos y, por tanto, comparte con ellos las características y tratamientos antes citados. 


\section{CuAdro IV}

LOS RESIDUOS PELIGROSOS EN LAS COMUNIDADES AUTÓNOMAS ESPAÑOLAS, SEGÚN ORDEN DE PRODUCCIÓN TOTAL

\begin{tabular}{l|c|c|c|c}
\hline & $\begin{array}{c}\text { Total } \\
\text { (toneladas/ } \\
\text { año) }\end{array}$ & $\begin{array}{c}\text { Porcentaje sin } \\
\text { tratamiento } \\
\text { «in situ» }\end{array}$ & $\begin{array}{c}\text { Porcentaje con } \\
\text { tratamiento } \\
\text { «in situ» }\end{array}$ & $\begin{array}{c}\text { Porcentaje de } \\
\text { producción } \\
\text { sobre el total } \\
\text { nacional }\end{array}$ \\
\hline Cataluña & 816.000 & 80,02 & 19,98 & 24,04 \\
País Vasco & 538.000 & 36,64 & 63,36 & 15,85 \\
Asturias & 525.000 & 14,29 & 85,71 & 15,47 \\
Galicia & 490.800 & 17,48 & 82,52 & 14,46 \\
Murcia & 201.100 & 74,63 & 25,37 & 5,92 \\
Madrid & 155.000 & 100 & 0 & 4,57 \\
Andalucía & 135.523 & 100 & 0 & 3,99 \\
Valencia & 134.880 & 100 & 0 & 3,97 \\
Castilla-La Mancha & 105.600 & 52,65 & 0 & 3,11 \\
Castilla y León & 86.500 & 100 & 0 & 2,55 \\
Aragón & 74.800 & 100 & 63,36 & 1,58 \\
Navarra & 53.500 & 36,64 & 0 & 0,81 \\
Canarias & 27.500 & 100 & 0 & 0,66 \\
Cantabria & 22.500 & 100 & 0 & 0,33 \\
La Rioja & 11.150 & 100 & 0 & 0,32 \\
Baleares & 11.000 & 100 & 0 & 0,16 \\
Extremadura & 5.600 & 100 & 33,97 & 100 \\
\multicolumn{1}{|c|}{ TOTAL } & 394.353 & 66,03 & & 0 \\
\hline
\end{tabular}

Fuente: Plan Nacional de Residuos Peligrosos, 1995. Elaboración propia.

El principal efecto de los residuos industriales incontrolados, y especialmente de los clasificados como peligrosos, es la contaminación del suelo - a más largo plazo también las aguas subterráneas-. Muchos suelos afectados durante décadas por vertidos incontrolados han quedado inutilizados para otros usos rentables y socialmente necesarios (vivienda, ganadería, agricultura, recreación, etc.), con las elevadas pérdidas económicas que, por otra parte, ello conlleva. A este respecto podemos adelantar que las estimaciones oficiales recientes han logrado clasificar en España 18.142 puntos de actividad industrial potencialmente contaminante y 4.389 emplazamientos contaminados. A escala regional, Andalucía, Cataluña, País Vasco y Madrid, figuran a la cabeza de las Comunidades Autónomas con suelos contaminados por motivos industriales. Aunque la mayor parte de las industrias radicadas en suelo español son potencialmente contaminantes con independencia de su ta- 
maño, la verdad es que los residuos peligrosos en su mayoría son generados por un grupo reducido de sectores industriales (cuadro V), entre los que destacan la industria química, la fabricación de automóviles y la fabricación de productos metálicos. En consecuencia la localización concreta de éstos es un factor fundamental a la hora de interpretar la diferente aportación regional a la producción total de residuos, como también lo es, lógicamente, el grado general de desarrollo industrial alcanzado por cada autonomía. Con esta perspectiva deben leerse los resultados del cuadro IV, donde Cataluña, País Vasco, Asturias y Galicia encabezan la lista de regiones productoras, con la diferencia de que las dos primeras lo son en razón básicamente de su extenso tejido industrial (Alio y Bru, 1988), y las otras dos porque acogen a factorías puntuales pero generadoras de un gran volumen de residuos peligrosos (Ensidesa, Asturiana de Zinc, Inespal, ...). De cualquier modo es especialmente importante la gestión que se siga en este cuarteto regional, ya que por sí solo produce el $70 \%$ de los residuos peligrosos españoles.

Lamentablemente las estimaciones cuantitativas que recogen los cuadros precedentes han de ser tomadas como meros indicadores de un problema hoy vigente, y no como cifras de total fiabilidad. Esto es así porque uno de los grandes escollos de las administraciones competen-

Cuadro V

CONTRIBUCIÓN DE LOS DISTINTOS SECTORES

DE ACTIVIDAD A LA PRODUCCIÓN DE RESIDUOS PELIGROSOS EN ESPAÑA

\begin{tabular}{l|c}
\hline Sector de actividad & $\begin{array}{c}\text { Porcentaje sobre } \\
\text { la producción } \\
\text { total }\end{array}$ \\
\hline Industria química & 32,6 \\
Industria del automóvil & 11,2 \\
Industria metalúrgica & 10,2 \\
Alimentación y bebidas & 8,1 \\
Industria papelera & 7,6 \\
Industria del cuero & 7,1 \\
Equipo eléctrico & 3,4 \\
Otras & 19,8 \\
\hline
\end{tabular}

Fuente: Plan Nacional de Residuos Peligrosos, 1995. Elaboración propia. 
tes viene siendo la dificultad en censar los residuos peligrosos que se crean (Alio, 1991), desde el momento en que los productores no cumplen - en la gran mayoría de los casos - con su obligación de efectuar la correspondiente declaración anual. En apartados sucesivos tendremos ocasión de referirnos a este grave obstáculo y al Plan Nacional de Residuos Peligrosos (1995-2000).

Por otra parte, conviene no olvidar que el poder contaminante de las sustancias consideradas tóxicas y peligrosas hace necesario contar con una vigilancia especial, y unos sistemas específicos de recogida, tratamiento y eliminación o depósito. El desfase existente entre este presupuesto teórico y su aplicación en la práctica es hoy otro de los grandes problemas ambientales de España. El dato de referencia es espeluznante: sólo reciben algún tipo de tratamiento alrededor de la tercera parte de los residuos peligros censados — que además son la minoría de los existentes-o, lo que es igual, al menos dos millones de toneladas de este tipo de sustancias se vierten en España sin control ni tratamiento alguno.

Pero, como casi siempre sucede en materia de problemas medioambientales, la interpretación de las cifras se presta a una doble lectura: la negativa o pesimista, que se sustenta en el último dato aportado, y la positiva o esperanzadora que confía en una paulatina resolución del problema basándose, con razón, en que la situación actual es notablemente mejor a la de hace diez años, cuando el porcentaje de residuos peligrosos sin controlar ascendía al $87 \%$.

En cualquier caso nos encontramos frente a una cuestión verdaderamente preocupante que, por ende, se ha visto agudizada a raíz de la promulgación de una reciente Directiva de la U.E. sobre instalaciones de vertido (1994), en virtud de la cual es posible que más de la mitad de los actuales vertederos españoles de este tipo de residuos quede fuera del marco legal, al menos hasta que se ejecuten las multimillonarias mejoras necesarias para adaptarlos a los nuevos requisitos exigidos.

Frustración y disparidad regional en la gestión de los residuos urbanos e industriales (1975-95)

La situación actual de los residuos urbanos e industriales no es todo lo halagüeña que cabría esperar, como se desprende, entre otros in- 
dicadores, de los altos porcentajes de vertidos incontrolados a que antes nos referíamos; a pesar de ello se han alcanzado algunos logros indudables, puesto que las referencias de dos décadas atrás eran realmente pésimas (Martín-Cobos et al., 1983). A continuación sintetizaremos las principales estrategias aplicadas en la gestión de los residuos en España, haciendo hincapié en los avances logrados, en las desigualdades regionales detectadas y en las grandes carencias que hoy se mantienen.

Actuaciones encaminadas a lograr un mayor control de los residuos generados. - En este apartado hay que diferenciar claramente entre las iniciativas que se centran en los residuos sólidos y urbanos, cuyo alcance ha sido globalmente satisfactorio, y las encauzadas hacia los residuos industriales, mucho menos efectivas hasta el momento.

Residuos Sólidos Urbanos.-A pesar de la anterior valoración, la incidencia de estas acciones ha sido muy desigual en el territorio español, en parte porque las competencias en la materia se encuentran repartidas entre diferentes administraciones, y ello se ha traducido en gestiones dispares (Rodríguez y Ruiz, 1983). No obstante, y con carácter general, las principales actuaciones en el terreno que nos ocupa - el control de los vertidos - han consistido, por un lado, en considerables inversiones para la adquisición de equipos de recogida y transporte de residuos, y para la instalación de vertederos sanitariamente controlados, y, por otro, en la adopción de un conjunto de medidas planificadoras entre las que sobresalen la redacción de Planes Directores de Gestión de Residuos Sólidos (P.D.R.S.) para ámbitos territoriales concretos ${ }^{4}$.

La Administración Central, al amparo de la «Ley de Recogida y Tratamiento de Residuos Sólidos Urbanos de 1975» —modificada por el Real Decreto 1.163/1986 - , estableció desde el año 1981 una partida presupuestaria concreta para subvencionar los proyectos tendentes a corregir la gestión de los residuos en todo el territorio español; asimismo, facilitó las dotaciones de equipo, vertederos y planes directores en aquellas zonas con mayores problemas. Posteriormente esta función ha pasado a ser competencia de las diferentes Comunidades Autónomas,

${ }^{4}$ La construcción e instalación de vertederos controlados se ha convertido en un grave y generalizado problema, debido a la escasez de suelo a una distancia razonable y a la abierta oposición de la población residente en las proximidades. 
que han continuado en esa línea. Los resultados de estas operaciones, ejecutadas mayoritariamente a partir de los años ochenta, pueden valorarse considerando algunas cifras:

a) En 1984 se generaron 10 millones de toneladas de R.S.U., de las que el $51 \%$-algo más de cinco millones de toneladas estuvieron controladas. En 1992 la producción fue de 13,8 millones de toneladas, ascendiendo el porcentaje anterior a $66 \%$, pero, debido al incremento en la producción global, aún permanecían 4,6 millones de toneladas de residuos fuera de control. Finalmente las última cifras disponibles manifiestan un paso sustancial en este terreno, ya que, si bien la producción ha mantenido su crecimiento sostenido (14,9 millones de toneladas), el control ha aumentado a un ritmo mayor hasta alcanzar el $82 \%$ de la misma, lo que equivale a decir que los residuos incontrolados han descendido hoy hasta los 2,6 millones de toneladas, como tuvimos ocasión de observar en el cuadro III.

b) Aunque cuantitativamente el progreso en el control de los residuos ha sido significativo, desde el punto de vista cualitativo ese avance no ha sido tan evidente. A pesar de haberse controlado la mayor parte de las basuras urbanas, lo cierto es que básicamente continúan teniendo como destino final los diferentes vertederos, puesto que el porcentaje de residuos reservados a la incineración se mantiene hoy por debajo del $5 \%$ y el de compostaje no sobrepasa el $14 \%$, unas proporciones incluso inferiores a las de hace una década (cuadro I).

c) Independientemente de lo señalado en relación con las cifras globales, si descendemos a la escala de las Comunidades Autónomas nos encontramos con interesantes contrastes. La desigualdad vuelve a ser notoria (cuadro III), lo cual, en nuestra opinión, es la manifestación básica y evidente de la diversidad de derroteros que sigue el panorama medioambiental español. El asunto es complejo y las razones que pueden aducirse para explicar diferencias tan radicales son también numerosas: nivel de renta, signo político de los poderes autonómicos y locales, tradición y capacidad en la gestión de residuos, nivel cultural medio de los residentes y sensibilidad ambiental de los mismos... Posiblemente distintas combinaciones de estos ingredientes justifiquen que, por ejemplo, en materia de residuos incontrolados, algunas autonomías superen el $18 \%$ de media española hasta niveles disparatados: Ceuta (100\%), Galicia (67 \%), Castilla-La Mancha (59 \%), Extremadura 
(56\%), Murcia (41\%)... En definitiva, las disparidades existentes son de tal calibre que invalidan los diagnósticos basados en promedios, que con tanta frecuencia últimamente se esgrimen en nuestro país como prueba de una gestión integrada y armónica, cuando en realidad el control de los residuos sólo se encuentra próximo a estar resuelto en algunas regiones concretas - léanse Cataluña, Madrid, Cantabria, Asturias y La Rioja-.

No obstante, con independencia de las combinaciones factoriales posibles, es indudable que estamos, ante todo, frente a un problema de desigual capacidad de planificación y gestión de unas Comunidades $\mathrm{Au}$ tónomas a otras, e incluso de unas provincias a otras, pero también es evidente y decisivo el desequilibrio de recursos económicos a favor de ciertas regiones para llevar a cabo las actuaciones necesarias - lo cual tiene mucho que ver con la óptima valoración de Madrid y Cataluña en la materia-. A tales escollos se añade en algunos ámbitos concretos la existencia de un fuerte poblamiento disperso que contribuye a dificultar las actuaciones de recogida, transporte y tratamiento de los R.S.U.; situaciones pésimas, como la gallega, posiblemente tengan mucho que ver con este hecho.

En definitiva, el estado de la cuestión puede quedar resumido en los siguientes términos: el esfuerzo en materia de control de R.S.U. ha sido considerable para el conjunto de España y las inversiones dirigidas a creación de centros de tratamiento se han multiplicado en los últimos años (cuadro VI). Sin embargo, persisten graves carencias, entre otras razones porque el problema de los residuos incontrolados - a pesar de haber disminuido su volumen- se caracteriza por una gran dispersión y por la existencia de agudos contrastes territoriales. Por esto, la cuestión clave hoy es la siguiente: el esfuerzo inversor de los últimos años desde las Administraciones central y autonómicas ha sido importante pero ¿ha tenido en cuenta la existencia de los grandes factores regionales diferenciadores a la hora de diseñar y ejecutar planes e inversiones? Creemos que no suficientemente.

Residuos industriales.-Dentro del grupo de los residuos industriales decíamos que sólo los considerados como tóxicos y peligrosos (R.T.P.) precisan de una gestión y control especiales, con tratamientos específicos y depósitos en vertederos de seguridad. La distribución de la producción de residuos industriales tóxicos y peligrosos en la geo- 


\section{CuAdro VI}

TIPO Y DISTRIBUCIÓN DE CENTROS DE TRATAMIENTO DE R.S.U. POR COMUNIDADES AUTÓNOMAS

\begin{tabular}{|c|c|c|c|c|}
\hline & $\begin{array}{l}\text { Número de } \\
\text { vertederos } \\
\text { controlados }\end{array}$ & $\begin{array}{l}\text { Número de } \\
\text { incinera- } \\
\text { doras }\end{array}$ & $\begin{array}{l}\text { Número de } \\
\text { plantas de } \\
\text { compostaje }\end{array}$ & $\begin{array}{c}\text { Total de } \\
\text { centros de } \\
\text { tratamiento }\end{array}$ \\
\hline Andalucía & 26 & 1 & 4 & 31 \\
\hline Aragón & 15 & - & - & 15 \\
\hline Asturias & 3 & - & - & 3 \\
\hline Baleares & 2 & - & 1 & 3 \\
\hline Canarias & 4 & 3 & 1 & 8 \\
\hline Cantabria & 2 & 4 & - & 6 \\
\hline Castilla-La Mancha & 4 & - & 1 & 5 \\
\hline Castilla y León & 27 & - & - & 27 \\
\hline Cataluña & 33 & 8 & 4 & 45 \\
\hline Extremadura & 5 & - & - & 5 \\
\hline Galicia & 6 & - & - & 6 \\
\hline Madrid & 6 & - & 2 & 8 \\
\hline Murcia & 一 & - & 4 & 4 \\
\hline Navarra & 14 & 2 & - & 16 \\
\hline País Vasco & 11 & 1 & - & 12 \\
\hline La Rioja & 4 & - & - & 4 \\
\hline Valencia & 12 & - & 9 & 21 \\
\hline Melilla & 1 & - & - & 1 \\
\hline Ceuta & - & - & - & 0 \\
\hline
\end{tabular}

Fuente: Medio Ambiente en España, 1996. (Ministerio de Medio Ambiente, 1997). Elaboración propia

grafía nacional varía de acuerdo con la implantación de la industria y la importancia relativa de las distintas actividades, como tuvimos ocasión de mostrar con anterioridad.

En general puede afirmarse que se ha progresado - aunque tímidamente- en el control de los R.T.P. a lo largo de la última década, en parte debido a la promulgación en 1986 de la Ley Básica sobre Residuos Tóxicos y Peligrosos. En 1984, antes de la entrada en vigor de la citada ley, la industria española producía 1,5 millones de toneladas anuales de residuos peligrosos, y sólo el $13 \%$ era objeto de algún tratamiento. El restante $87 \%$ se eliminaba de forma incontrolada, originando impactos ambientales de graves consecuencias para el aire, el suelo y el agua, a los que ya nos hemos referido. En aquel año España únicamente contaba 
con una planta de neutralización de ácidos en el País Vasco, y los aceites usados eran tratados casi en su totalidad por CAMPSA, que disponía de tres plantas para este fin.

Frente a tal situación, la Ley de Residuos Tóxicos y Peligrosos ha contribuido a regular legalmente el uso y vertido de los residuos industriales de mayor impacto, pero no ha conseguido el deseado control sobre los mismos. Como apoyo a ella, en 1989 se aprobó el denominado Plan Nacional de Residuos Industriales, con el que se pretendió «crear el marco adecuado para garantizar el tratamiento o eliminación de los residuos industriales especiales y fomentar las técnicas tendentes a reducir su generación». Sin embargo, pese a la puesta en marcha de este ambicioso plan, los resultados dejan mucho que desear, ya que las estimaciones oficiales existentes indican que la cifra de residuos tóxicos y peligrosos asciende en 1994 a 3,3 millones de toneladas por año, de las cuales el $66 \%$ se vierte aún sin control alguno. Es cierto, en definitiva, que ha aumentado notablemente el porcentaje de residuos controlados desde 1984, pero, al haber crecido igualmente su volumen, la situación sigue siendo grave. La causa de esto se encuentra, en gran medida, en la actitud de las empresas productoras de estos residuos, obligadas por el Reglamento vigente a entregar anualmente una declaración de residuos generados. De hecho, la Secretaría General de Medio Ambiente considera que el número de declaraciones que debería presentarse oscila en torno a 30.000 -incluyendo sólo a las empresas que originan más de $10.000 \mathrm{~kg}$ al año-y, frente a esta apreciación, en el año $1996 \mathrm{se}$ efectuaron en toda España 4.119 declaraciones, es decir, el $14 \%$ de las que legalmente deberían haberlo hecho.

Otro problema relacionado con los vertidos incontrolados de residuos peligrosos es el de sus importaciones y exportaciones, carentes de controles rigurosos hasta el año 1990, fecha de aprobación de una orden ministerial reguladora de estos tránsitos. Más tarde entró en vigor el Reglamento CEE n. ${ }^{\circ}$ 259/93, con la intención de resolver un problema general que, en el caso de España, supone unas importaciones próximas a 85.000 toneladas anuales, en tanto que las exportaciones han ido creciendo hasta superar las 20.000 toneladas en 1995. Los resultados comienzan a cosecharse, ya que las declaraciones de residuos peligrosos que atraviesan la frontera española se han multiplicado por siete desde 1989 (OCDE, 1997), aunque se estima muy importante el volumen de residuos que aún no se declara. 
En definitiva, deben quedar claros dos aspectos en relación con los residuos tóxicos y peligrosos. En primer lugar que ni la promulgación de una ley específica para su gestión, ni la puesta en marcha del citado Plan Nacional, han logrado por sí mismos una solución eficaz a un problema ya antiguo; en segundo término, y puesto que existen los instrumentos jurídicos necesarios, es imprescindible que las administraciones autonómicas exijan a las empresas productoras el cumplimiento de sus obligaciones con mayor empeño.

Políticas auspiciadoras del reciclado y la reutilización de los residuos.-Las iniciativas encaminadas al fomento del reciclado y la reutilización conforman una segunda gran línea de actuación en la gestión reciente de los residuos en España. A la vez, en el conjunto de las diversas operaciones tendentes a tal fin es conveniente destacar tres de ellas, por su significado cualitativo y cuantitativo:

a) La «recogida selectiva» de los residuos sólidos urbanos e industriales.

b) $\mathrm{El}$ «compostaje» de los residuos sólidos urbanos y asimilados.

c) La «regeneración» de los aceites minerales y sintéticos usados.

a) La recogida selectiva en España se ha centrado básicamente en el papel, el cartón, el vidrio y, últimamente, el plástico. Es cierto que se han obtenido resultados notables durante la última década, si bien queda un largo camino por recorrer.

Si nos detenemos en el papel y el cartón observaremos que se ha producido una evolución significativa aunque no suficiente: en 1984 se recuperaron 1.214.400 toneladas de papel y cartón usados, lo que equivale a decir que se ha evitado cortar la madera existente en 170.000 hectáreas arboladas; once años después se han alcanzado 2.116.600. toneladas, cifra de recuperación que equivale al $36 \%$ de lo que se consume en España, y que no obstante sitúa a España lejos del promedio de la U.E., donde ocupa el décimo lugar por este concepto. Este material usado se reutiliza junto con otras 572.900 toneladas importadas, de forma que es prioritario incrementar la tasa de recogida selectiva de este material, pues la demanda interna es hoy sustancialmente mayor a la capacidad de recogida y reutilización. Los beneficios económicos y ecológicos de un avance en esta línea serían considerables, pero para ello 
habrán de resolverse dos situaciones coyunturales desfavorables: la primera consiste en que la recogida y reciclado de papel usado en España ha recaído durante bastante tiempo en sectores empresariales privados sin apoyo oficial; la segunda es que estos sectores han perdido competitividad en el marco internacional, debido a la política de subvenciones que algunos países vienen aplicando a la recogida de papel usado, lo que ha tenido como consecuencia inmediata el descenso de los precios de este material en los mercados europeos. La intervención de la Administración española es necesaria, por tanto, y ha comenzado con una campaña de la Secretaría de Estado de Medio Ambiente en colaboración con las Comunidades Autónomas, para la instalación de contenedores específicos en espacios públicos de las ciudades españolas, así como la adquisición de camiones para la recogida selectiva de estos residuos. En principio la meta establecida consiste en alcanzar una dotación mínima de un contenedor para recogida de papel-cartón por cada 2.000 habitantes y sucesivamente llegar a 1.000 e incluso a 500 en casos puntuales (Ordóñez, 1994).

La recogida selectiva de vidrio doméstico es más reciente y se inició en 1982 en Madrid y Barcelona con la instalación de 236 puntos de depósito. Desde entonces la evolución ha sido creciente y positiva a tenor de los resultados obtenidos. En 1984 la red se extendía ya sustancialmente y se reunían 7.800 toneladas. Ocho años más tarde, en 1992, existían 13.500 contenedores, a través de los cuales se almacenaron 54.000 toneladas de vidrio, aunque el salto espectacular se produce en el trienio 92-95 cuando el número de contenedores instalados (34.956) casi se triplica, y el volumen de recogida se incrementa notablemente (165.943 toneladas). A estos resultados habría que sumar las 26.245 toneladas de vidrio doméstico obtenidas por otros medios y las 209.169 de vidrio industrial recuperado. Atendiendo a la situación respectiva de las Comunidades Autónomas se extraen interesantes diferencias, que tienen como trasfondo la dispar sensibilidad ambiental adquirida por parte de los ciudadanos, gestores autonómicos y locales; en primer lugar hay que destacar la óptima y sostenida gestión de Comunidades como Cataluña o el País Vasco, con unas tasas de recuperación - en relación con su peso demográfico- verdaderamente notables desde el comienzo. Asimismo resulta espectacular el salto cuantitativo logrado por otras regiones cuyo estado en 1992 era de franco retraso en relación a su potencial y que en un bienio han logrado alcanzar cotas de recuperación 
apropiadas; en este sentido sobresalen los incrementos recientes de Murcia (3.115\%), Canarias (2.200\%), Galicia (936\%), Castilla-La Mancha (853\%), Valencia (786 \%), Baleares (579\%) o Andalucía (446\%). No obstante en el extremo opuesto también se han producido casos de estancamiento y retroceso, como el de Asturias.

En definitiva la situación global ha mejorado sustancialmente y las perspectivas son favorables, puesto que el convenio Estado-Autonomías contempla vías de actuación infraestructural para recuperación del vidrio similares a las ejecutadas para el papel. Sin duda ello es necesario si pretendemos equiparar el nivel español (31\% de recuperación de vidrio doméstico e industrial) al europeo (42\%). Los alicientes para ello son suficientes y, entre otros, pensemos en el ahorro de más de 100 kilos de petróleo por cada tonelada de vidrio reciclado.

Los plásticos han ganado peso en el conjunto de los residuos españoles, entre los que ocupan ya un tercer lugar, situándose tras las materias citadas arriba (10\% del volumen de R.S.U.). No obstante su reciclado y reutilización ha avanzado poco en España, puesto que el $74 \%$ de la anterior cifra fue a parar a vertederos, sólo el $12 \%$ se recicló, el $8 \%$ se incineró con recuperación de energía, y el $6 \%$ restante se incineró sin recuperación energética. Obviamente el primer asunto a resolver ha de consistir en alcanzar una distribución distinta de tales porcentajes, pero además existe otro escollo en vías de solución. Nos estamos refiriendo al problema de la importación incontrolada de residuos plásticos desde otros países que, como Alemania, ofrecen incentivos económicos a quienes se hagan cargo de ellos. Este tipo de importación ha dado lugar a acumulaciones notables en vertederos o almacenamientos irregulares, por ejemplo en algunos puntos concretos de las Comunidades de Madrid y Aragón, donde - una vez cobrados los incentivos citados- no se había previsto reciclado ni tratamiento alguno (Martin, 1994). En definitiva, hasta el momento las iniciativas pioneras en España para la recogida selectiva de residuos plásticos se caracterizan por su dispersión y heterogeneidad, unas insuficiencias que deben ser corregidas a corto plazo si se desea amortiguar el impacto que supondrá el gran crecimiento previsto en la utilización de los diversos tipos de plásticos.

b) Junto a la recogida selectiva hay que señalar también la importante función que desempeñan en la actualidad las plantas de compostaje. El compost se obtiene a través de la fermentación controlada de la parte orgánica de los residuos urbanos y de los industriales asimilados a 
aquéllos, y el producto resultante es un regenerador de suelos. Su aprovechamiento puede tener una transcendencia singular en países que, como España, presentan graves deficiencias edáficas de materia orgánica. Por ello la mayoría de las instalaciones están ubicadas en las regiones levantinas y meridionales, en virtud de la considerable extensión de su tierras agrícolas. E incluso, si la demanda agrícola no fuera suficiente, se podría emplear en la recuperación de tierras quemadas o desertizadas, y en los suelos con masas forestales, siempre y cuando los costes de generación, tratamiento y manipulación, transporte, etc. lo hicieran viable.

En 1995, en el conjunto de las plantas de compostaje se han tratado 2.086.347 toneladas de basura de las que se han conseguido casi 360.000 toneladas de compost, es decir, un rendimiento aproximado del $17 \%$. Pero, además, el proceso del compostaje produce unos efectos positivos indirectos, porque permite la recuperación de papel, vidrio, plástico y chatarra que, previamente, han de separarse de la materia orgánica útil. Las cifras de productos recuperados no son desdeñables en modo alguno, puesto que durante el citado año se han obtenido 79.416 toneladas de las materias citadas en las plantas españolas de compost.

c) Por último, en el marco del reciclado y la reutilización es también interesante la orientación dada a los aceites minerales usados en los últimos años. Como tales se consideran todos los aceites industriales con base mineral o sintética y lubricantes que se hayan vuelto inadecuados para el uso que se les hubiere asignado inicialmente, en particular los aceites utilizados de los motores de combustión y de los sistemas de transmisión, turbinas y sistemas hidráulicos. La situación de estos productos es ciertamente preocupante en España. Por ejemplo, está bastante extendida, a nivel particular, la práctica de cambiar personalmente el aceite al propio coche y verter el usado en el suelo, dando lugar a daños ambientales considerables si este residuo se filtra a las conducciones de agua próximas, entre otros riesgos. También a escala industrial plantea numerosos problemas, tanto en lo referido a su regeneración como a su eliminación: los contenedores metálicos pueden verse afectados por la corrosión, y asimismo existen aún numerosos vertidos incontrolados a cursos de agua o vertederos sin protección, habiéndose detectado afecciones graves en el conjunto de los recursos naturales (suelo, agua, flora, fauna, etc.), que pueden concluir con la inutilización de espacios para otros usos y favorecer procesos de desertización. 
La mejora en la gestión de estos residuos ha sido considerable, aunque los resultados sólo son sustanciales en lo referido al control de los aceites usados y no así a su regeneración. En 1992 se controlaron en España unas 40.000 toneladas, pero un volumen indeterminado fue vertido sin control; en 1995 la cifra de residuos recogidos fue ya de 114.000 toneladas, pero de éstas sólo 4.000 se destinaron a regeneración en varias plantas existentes en España - especialmente en las tres de Cataluña, Madrid y Valencia- y el resto se orientó a la combustión con recuperación energética en cementeras, ladrilleras y plantas de cerámica ubicadas en Galicia, Madrid, Andalucía, Murcia, La Rioja y Navarra principalmente. La Administración central subvenciona a las Comunidades Autónomas para la gestión de sus aceites usados y, sin embargo, las cifras de regeneración expuestas anteriormente no son aceptables. En consecuencia, se pretende invertir la tendencia a medio plazo, con el apoyo, entre otras acciones, de una Ley de Aceites Usados específica.

Medidas específicas para el saneamiento de suelos contaminados por residuos industriales peligrosos. - A las políticas de control, reciclado y reutilización de residuos que hemos descrito se ha sumado recientemente otra dirigida a localizar, analizar y sanear suelos contaminados por residuos peligrosos. Estos han sido en buena medida depositados sin tratamiento alguno y han alterado sustancialmente los componentes naturales de la capa edáfica en las áreas de vertido, dando lugar a consecuencias de diversa índole sobre el conjunto de los seres vivos, incluido el hombre, que puede verse afectado a través del uso de acuíferos contaminados o de la ingestión de especies vegetales desarrolladas en un sustrato alterado.

En 1991 el MOPTMA inició un Inventario Nacional de Suelos Contaminados, de entre cuyos resultados creemos interesante destacar lo siguiente:

1. $^{\circ} \quad$ Se han identificado en España un total de 4.589 emplazamientos potencialmente contaminados. Es ésta una situación preocupante pero desgraciadamente muy extendida en nuestro entorno europeo: Alemania reconoce la existencia de 143.000 emplazamientos contaminados, y en la misma línea se encuentran Holanda (100.000), Austria (24.000) o Dinamarca (10.000), entre otros. 
2..$^{\circ}$ Se estima que hay 18.142 establecimientos industriales que, a tenor de sus características, constituyen focos potenciales de contaminación del suelo en su entorno.

$3 .^{\circ}$ Se ha estudiado en detalle una muestra de 249 emplazamientos alterados, extrayéndose entre otras, estas conclusiones:

- La práctica totalidad no cumplen las exigencias legales establecidas; entre las irregularidades destaca que el acceso sea libre al $60 \%$ de ellos.

- El riesgo de afección a las aguas es muy alto. Se calcula que el $60 \%$ pueden afectar a las aguas subterráneas y el $50 \%$ a las superficiales.

\section{CuAdRo VII}

INVENTARIO DE ACTIVIDADES INDUSTRIALES Y DE DESPLAZAMIENTOS POTENCIALMENTE CONTAMINADOS EN LAS COMUNIDADES AUTÓNOMAS

\begin{tabular}{l|c|c}
\hline & $\begin{array}{c}\text { Número de } \\
\text { actividades } \\
\text { industriales }\end{array}$ & $\begin{array}{c}\text { número de } \\
\text { emplazamientos } \\
\text { potencialmente } \\
\text { contaminados }\end{array}$ \\
\hline Andalucía & 1.395 & 604 \\
Aragón & 717 & 321 \\
Asturias & 394 & 156 \\
Baleares & 303 & 23 \\
Canarias & 396 & 217 \\
Cantabria & 238 & 81 \\
Castilla y León & 811 & 383 \\
Castilla-La Mancha & 287 & 252 \\
Cataluña & 4.913 & 569 \\
Extremadura & 183 & 46 \\
Galicia & 860 & 491 \\
Madrid & 2.277 & 222 \\
Murcia & 469 & 75 \\
Navarra & 334 & 22 \\
País Vasco & 2.059 & 538 \\
La Rioja & 153 & 35 \\
Valencia & 2.330 & 350 \\
Ceuta y Melilla & 22 & 4 \\
\multicolumn{1}{c|}{ TOTAL } & 18.142 & 4.389 \\
\hline
\end{tabular}

Fuente: Programa de Recuperación de Suelos Contaminados. M.O.P.T.M.A., 1993. 
- La gran mayoría de los suelos contaminados están situados dentro de cascos urbanos o a menos de 200 metros de los mismos.

- Los contaminantes más frecuentes son diversos metales pesados, aceites minerales, hidrocarburos y -con menor frecuencia pero mayor toxicidad-arsénico y mercurio.

Andalucía, Cataluña, País Vasco y Galicia figuran a la cabeza de las Comunidades Autónomas con suelos contaminados por causa industrial; en cada una de ellas se han inventariado más de 500 emplazamientos virtualmente contaminados (cuadro VII) ${ }^{5}$. La constatación de la magnitud de este problema ambiental ha desembocado, entre otras cosas, en el diseño de un Plan Nacional de Recuperación de Suelos Contaminados; en el apartado correspondiente haremos alusión a lo esencial de esta nueva estrategia.

A la búsqueda de alternativas para un grupo heterogéneo de residuos específicos.-Por sus características específicas algunos residuos se diferencian claramente del flujo general tratado en apartados anteriores; su heterogeneidad es tal que dificulta enormemente la gestión y la consecución de alternativas satisfactorias. Sobre todos ellos planean, como escollos a superar, la necesidad de implicar a los sectores sociales y empresariales en la búsqueda de soluciones, las limitaciones presupuestarias y la urgencia en lograr nuevas tecnologías que faciliten su posible reutilización, su eliminación o su depósito final en los casos pertinentes. Residuos radiactivos, residuos biosanitarios especiales, pilas, neumáticos, aparatos eléctricos y electrónicos desechados, etc. son algunos de los que mayor atención suscitan en el momento actual, a los que se sumarán, a corto plazo, otros productos diversos. Lamentablemente, hasta el momento, la actuación sobre este heterogéneo grupo ha estado más volcada en la planificación y búsqueda de soluciones que en la resolución de los problemas concretos.

$\mathrm{El}$ futuro de los residuos radiactivos está envuelto en la polémica. Sin entrar en consideraciones subjetivas sobre el riesgo que supone la presencia de una amplia red de centrales nucleares en nuestro país y la dependencia que de ellas se tiene - generan aproximadamente una

\footnotetext{
${ }^{5}$ Resulta cuando menos paradójico que Andalucía, no siendo unas de las grandes productoras de residuos peligrosos, encabece la relación regional de emplazamientos contaminados.
} 
cuarta parte de la energía eléctrica producida en España-, no debemos obviar la gran dificultad que hoy plantea esta fuente de energía, es decir, el incierto destino de los residuos que engendra, sobre todo porque España es el décimo productor mundial de los mismos. En el momento actual estamos ante un asunto sin resolver, a pesar de la labor desarrollada por la empresa nacional ENRESA - encargada de su gestión desde 1985- entre cuyas actuaciones principales destaca la consolidación de las instalaciones de El Cabril (Hornachuelos, Córdoba) como depósito general de los residuos de baja actividad que se producen en España. Sin embargo las soluciones para los residuos de alta actividad, de un enorme riesgo, no se vislumbran hasta el momento, y su producción continúa creciendo; entre las arriesgadas alternativas que se contemplan destaca el enterramiento profundo en estructuras geológicas de gran estabilidad (granito, sal, etc.).

Como residuos biosanitarios especiales (R.B.E.) conocemos aquella fracción de las 200.000 toneladas de residuos sanitarios clasificada como tóxica y peligrosa, de forma que comparte la problemática referida arriba para los residuos peligrosos, aunque por su particular origen y composición merece tratamiento aparte. En España la producción de R.B.E. se cifra en unas 46.000 toneladas/año, que vienen siendo gestionadas de manera verdaderamente deficiente, desde el momento en que no son objeto de manipulación especial, predominando una incineración masiva y escasamente controlada. A medio plazo se contemplan diversas alternativas para ellos en el nuevo Plan Nacional de Residuos Peligrosos que abordaremos posteriormente.

Tampoco es baladí el problema de los neumáticos usados, de los que España origina anualmente más de 150.000 toneladas, una modesta aportación a un total europeo que sobrepasa los dos millones. Aunque no podemos detenernos en ellas, las consecuencias tanto de su almacenamiento como de su incineración masiva son muy preocupantes. Estas ya se conocen suficientemente, por lo que desde la Unión Europea se impulsó en 1991 la creación de un grupo de trabajo específico para tal fin, sobre todo porque las previsiones de crecimiento de este residuo son evidentes. La misión de este grupo es variada: se trata de controlar el residuo, procurar su reducción, y especialmente fomentar su reutilización y reciclaje, aprovechando sus múltiples aplicaciones y su alta capacidad energética. Desde aquel año las reuniones se mantienen y se busca la necesaria colaboración de fabricantes, distribuidores, recauchutadores y 
tratadores, junto con las distintas administraciones. Como sucede con casi todos los macroplanes medioambientales, el que se está diseñando para los neumáticos también rebosa «optimismo», pues no podemos otorgar otro calificativo más prudente a la intención de la Unión Europea de haber logrado el aprovechamiento total de estos residuos en el año 2002.

En la misma línea de debate, a través de grupos de trabajo promovidos por la Unión Europea, se encuentran los residuos de aparatos eléctricos y electrónicos, e igualmente la pretensión básica del planificador consiste en implicar en la búsqueda de soluciones a todos los agentes privados que intervienen en el ciclo de vida del aparato, para así acelerar la introducción de nuevas tecnologías y productos más limpios, que faciliten la reutilización, recuperación o eliminación en los casos necesarios.

Por otra parte, la gestión de pilas y acumuladores que contienen cadmio y mercurio se encuentra más avanzada, puesto que está en marcha la campaña de colaboración emprendida por el MOPTMA con la Confederación Española de Cajas de Ahorros (C.E.C.A.) para recogida de pilas botón, que son finalmente depositadas en la planta de S. Fernando de Henares (Madrid), a la que se sumará una planta de tratamiento en Somozas (La Coruña), así como otras varias promovidas por algunas autonomías que han emprendido sistemas propios de recogida de pilas en sus respectivos territorios (Valencia, Cataluña y País Vasco).

\section{Las grandes actuaciones en marcha o la imposible} obtención de resultados espectaculares a corto plazo

Los resultados de las actuaciones dirigidas a resolver el problema de los residuos —diseñadas mayoritariamente en el período 1980-95- no han sido los pronosticados en su día. Esta conclusión es especialmente clara en lo concerniente a los residuos tóxicos y peligrosos, pero también se han frustrado parcialmente las previsiones de control y tratamiento de residuos sólidos urbanos establecidas entonces. El reconocimiento, en unos casos, de intervenciones insuficientes y, en otros, de actuaciones mal diseñadas y ejecutadas, ha sido el principal motivo de un rediseño de la política española de residuos con el horizonte del próximo milenio. 
Las nuevas líneas de trabajo recogen las directrices comunitarias plasmadas en la «Estrategia de Gestión de Residuos» (1989) de la Comisión de las Comunidades Europeas, sobre todo en lo concerniente a la potenciación de la prevención - especialmente en la industria: nuevos sistemas de envasado, aplicación de tecnologías más limpias, etc. y del reciclado, que requiere grandes esfuerzos hasta conseguir que el residuo se clasifique en el mismo lugar donde se produce, que se generalicen los contenedores apropiados, y que se consoliden servicios de recogida especializados. A corto plazo se pretenden alcanzar logros espectaculares a través de la ejecución de diversos macroplanes a escala estatal que pasamos a describir.

El Plan Nacional de Residuos Peligrosos (1995-2000)._En parte este documento planificador es la consecuencia lógica del fracaso del Plan Nacional de Residuos Tóxicos y Peligrosos de 1989, que tan esperanzador se presentó en el momento de su aprobación y que tantos escollos ha encontrado a lo largo de su vigencia, especialmente de tipo financiero, de rechazo social y de discrepancia política. Por ejemplo, la ubicación adecuada de las plantas de tratamiento proyectadas, de acuerdo con criterios de máxima rentabilidad y proximidad a centros de generación - piedra angular del Plan- no ha podido llevarse a cabo ante la imposibilidad de armonizar intereses políticos, sociales y empresariales (Fundación MAPFRE, 1994)

No obstante, el nuevo Plan en marcha no debe ser entendido como un mero continuador de aquél, puesto que recoge renovadas orientaciones emanadas de las directivas comunitarias más recientes, en virtud de las cuales ahora se establecen tres grandes prioridades:

a) Reducción en origen de la cantidad de residuos peligrosos. Se trata de una estrategia no contemplada con suficiente énfasis en políticas anteriores y que pretende alcanzar unas metas tan ambiciosas que, en el mejor de los casos, han sido calificadas de «irreales», a tenor de las dificultades enormes que deben superarse para lograr la prevista reducción en origen del $40 \%$ de los residuos peligrosos hoy generados. Así, en opinión del Club Español de los Residuos (fundado en 1994), el porcentaje de reducción en el quinquenio previsto estará situado como máximo entre el 10 y el $20 \%$.

Reducir la generación de residuos en la industria es uno de los ejes 
centrales de la nueva política, partiendo de la tesis de que generar menos residuos no sólo reporta beneficios al medio ambiente, sino que también es rentable para la propia empresa, a la que supondrá un ahorro evidente todo lo que signifique recortar los costes ligados al manejo de los desechos que hoy origina. En realidad se intenta difundir entre el empresariado la idea de que en la mayor parte de los sectores industriales es técnicamente posible corregir problemas medioambientales y reducir producción de residuos mediante el uso de tecnologías más limpias. Este cambio tecnológico iría también acompañado de una reducción de costos derivada del ahorro en agua, energía, materias primas, etc. En esta línea se centra el trabajo de la sociedad estatal EMGRISA (empresa para la gestión de residuos industriales) que está realizando estudios específicos sobre sectores industriales concretos (siderurgia integral y acerías; refino de petróleo; curtidos; química orgánica, etc.), tendentes a demostrar la viabilidad de los anteriores presupuestos teóricos y a concretar las vías a seguir en tales sectores para poder cumplir los ambiciosos objetivos del nuevo Plan Nacional de Residuos Peligrosos.

b) Fomento del reciclaje y la reutilización. Esta alternativa complementa a la anterior y adolece, como ella, de excesivo optimismo, sobre todo si reconocemos la escasa tradición española en el reciclaje de residuos peligrosos y la mínima infraestructura existente hasta la fecha. En este caso se quiere alcanzar un porcentaje de reciclado y reutilización del $20 \%$ del volumen actual de residuos, con la pretensión de que la mayor parte de ellos sean tratados y reutilizados en el propio complejo industrial que los genera, eliminando así los gastos y los riesgos que conlleva el traslado de sustancias peligrosas, y abriendo la posibilidad de reutilizar en los procesos industriales aquellos residuos compatibles con los mismos.

c) Tratamiento ambiental adecuado para los residuos generados. En nuestra opinión bajo este enunciado del Plan se recoge el conjunto de pretensiones que cualquier país quisiera lograr a corto plazo, y que se incluyen automáticamente en todo macroplan que se precie, aún a sabiendas de la imposibilidad de lograrlas. Esto es obvio, y basta para convencerse con recordar el $66 \%$ de los residuos peligrosos que se vierten de forma incontrolada en España. Sin embargo, el Plan es claro al respecto cuando establece que en el año 2000 estará gararantizado el acceso de todos los residuos peligrosos a infraestructuras de tratamiento ade- 
cuadas, y que deberá estar disponible, además, una «red supraautonómica» de depósitos de seguridad, donde albergar todos aquellos residuos que no hayan podido ser tratados por otra vía. ¿Hipocresía?, ¿desconocimiento?, ¿intento de mejorar nuestra imagen en el seno de la U.E.?, ¿estrategia esperanzadora?..., ciertamente es complicado interpretar lo que el planificador piensa cuando establece pretensiones tan desproporcionadas.

Para la consecución de estos grandes objetivos el Plan diseña un conjunto de incentivos fiscales, financieros y legislativos a aplicar mediante convenios entre el Estado, las Comunidades Autónomas y las empresas implicadas. Las primeras estimaciones evalúan en 180.000 millones de pesetas la inversión necesaria, cantidad que deberá provenir en parte de los Fondos de Cohesión comunitarios.

El Plan Nacional de Recuperación de Suelos Contaminados (19952005).-La necesidad de emprender medidas a corto plazo dirigidas a recuperar suelos alterados a consecuencia de vertidos incontrolados es una consecuencia evidente de los resultados del Inventario Nacional de Suelos Contaminados que comentábamos en páginas anteriores. No obstante, este Plan Nacional aspira a ser exclusivamente un instrumento coordinador e impulsor - mediante la vía financiera- de las actuaciones de las Comunidades Autónomas, puesto que éstas tienen asignadas las competencias para actuar en los suelos contaminados de sus respectivos territorios.

La estrategia fijada en el documento consiste en actuar en 274 zonas especialmente conflictivas, seleccionadas entre los más de 4.000 emplazamientos contaminados que el Inventario recoge; además se dará carácter urgente a las 61 que presentan un estado más grave. La inversión prevista para el decenio de vigencia asciende aproximadamente a 130.000 millones de pesetas, de los cuales el Ministerio de Medio Ambiente aportará el 50\%. Hay que tener presente el respaldo claro de la Unión Europea a este tipo de acciones, ya que, a través de los Fondos de Cohesión y FEDER, financiará hasta el 80\% de algunas actuaciones concretas.

El Plan Nacional de Residuos Sólidos Urbanos (1995-2005).-La redacción de un Plan Nacional de Residuos Sólidos Urbanos está encomendada a la Administración central por el Real Decreto Legislativo 
1.163/1986, que modificó a la Ley 421/1975 sobre desechos y residuos sólidos urbanos. La tardanza en su elaboración y aprobación - a pesar de haberse fijado como período de aplicación el decenio 1995/2005- es buena prueba de la confusión que rodea al problema de los residuos en España. Una vez concluido, el Plan debería convertirse en el instrumento marco de referencia en la gestión estatal de este tipo de residuos, diseñando sus grandes líneas de actuación a partir de la información recogida en los Planes Directores de Gestión de Residuos Sólidos de las respectivas Comunidades Autónomas y otros ámbitos territoriales concretos, que se han venido elaborando desde la promulgación de la ley marco de 1975. En tales documentos se refleja una síntesis de la situación de todos los capítulos básicos del proceso de gestión de residuos (producción, sistemas de recogida, recuperación, tratamiento y eliminación, etc.) desagregados por grandes municipios, comarcas o mancomunidades.

Una vez evaluado el estado global de los R.S.U. en el territorio nacional, el plan fijará las actuaciones e inversiones estructurales a realizar con un doble horizonte en los años 2000 y 2005, y dará prioridad a potenciar la minimización, recuperación y reciclaje de los residuos, su eliminación segura y la restauración de zonas degradadas por vertidos incorrectos de los mismos, todo ello a partir de la incorporación de toda la normativa en vigor tanto nacional como comunitaria.

\section{Perspectivas para el problema de los residuos urbanos e industriales}

El futuro del problema de los residuos es ciertamente complejo y las alternativas clásicas para su eliminación se tornan cada vez menos efectivas. Por ejemplo, la construcción de vertederos y plantas de tratamiento choca con grandes obstáculos en muchas ciudades, debido a la escasez de suelo a una distancia razonable de los cascos urbanos o a la firme oposición de los residentes; la reutilización y el reciclado de los residuos son estrategias prometedoras pero aún germinales; la incineración está presidida por la polémica en torno a sus emisiones tóxicas y la dificultad de encontrar emplazamientos adecuados para la instalación de plantas incineradoras, un problema éste que es especialmente complejo en España. En un reciente trabajo de Tapia y Toharia (1996) se 
insiste en que la incineración en España ha sido un procedimiento sacrificado por la Administración en aras de la concordia con los grupos ecologistas, en un momento - aclaran- en el que en Europa y resto del mundo desarrollado no sólo se sigue incinerando sino que se están incrementando estas instalaciones, renovando lógicamente los sistemas de combustión y reduciendo al mínimo las emisiones. Para estos autores es evidente que hay residuos no aptos para el tratamiento físico-químico, poco adecuados para el almacenamiento y que, además, poseen un alto contenido energético, por lo tanto se preguntan: ¿es tan descabellado promocionar la combustión controlada de residuos con producción energética secundaria?

Y, en este contexto, el volumen de los residuos continua creciendo en nuestro país, y las carencias tradicionales, demostradas a lo largo del análisis, se van solventando lentamente y con extrema desigualdad regional. Un reciente informe de la OCDE (1997) sobre los resultados medioambientales en España viene a reforzar lo dicho, cuando en sus conclusiones recoge la siguiente reflexión:

«Los resultados concretos se han limitado hasta la fecha a algunos progresos en el aspecto de la eliminación y del reciclado de los residuos. Poco se ha progresado en la prevención de la producción de residuos. Es urgente mejorar la gestión de los residuos peligrosos: las capacidades de tratamiento y eliminación son insuficientes, por lo cual son propicias para las exportaciones y la eliminación descontrolada (...) España deberá progresar en todos los niveles de la jerarquía de los residuos. La mejora de la gestión de residuos en las Comunidades Autónomas representa otro objetivo importante: algunas comunidades no disponen de las suficientes capacidades para controlar los residuos peligrosos y, en algunas regiones, los vertederos descontrolados representan más de la mitad del volumen de los residuos sólidos» (OCDE, 1997, 27)

La realidad descrita está llevando a un replanteamiento de la gestión tradicional de las basuras, mediante el cual se pretende ahora apostar por soluciones que, lejos de ser novedosas, se vienen barajando desde hace décadas (Ayres y Kneese, 1973). Conceptos como los de prevención o reducción en origen, reciclado y reintroducción de los residuos en los procesos productivos, se han postulado hace largo tiempo, pero siempre advirtiéndose de las dificultades que su consecución plantea. Es cierto que la mayoría de los residuos, a un cierto coste, pueden recuperarse para fines útiles, pero también lo es que con frecuencia el coste que im- 
plica la recuperación es mayor que el que supone obtener nuevas materias primas de la naturaleza y eliminar los residuos resultantes por los procedimientos tradicionales, una alternativa a la que difícilmente se sustraen las empresas que se rigen por cuentas de resultados. El reconocimiento de esto explica el énfasis puesto ahora en la necesidad de incentivar económicamente a los productores y gestores de los residuos urbanos e industriales - por ejemplo en los grandes planes antes descritos- como la mejor solución para que se sumen a las nuevas directrices. Pero incluso suponiendo que se alcance la necesaria colaboración política, social y empresarial, y que se superen los escollos técnicos existentes, ¿podrá el sector público a medio plazo asumir los costes que implica una gestión de los residuos altamente subvencionada?

Parece que las cuentas no cuadran, y que la solución a medio plazo se busca en la creación de «impuestos verdes» como los contemplados en las futuras leyes sobre aceites usados y sobre envases y residuos de envases. Desde luego en este sentido apuntan las recomendaciones de la OCDE (1997) a la Administración española, cuando recientemente le ha sugerido la utilización de una serie de instrumentos fiscales para propiciar la minimización de los residuos, tales como tarifas aplicables a la recogida de basuras, tarifas para su descarga en vertederos, tarifas aplicables a los productos, sistemas de depósito y devolución, etc. Finalmente, a la vez que se consideran las anteriores propuestas, desde el nuevo Ministerio de Medio Ambiente se trabaja en la elaboración de una Ley Básica de Residuos para intentar ordenar el panorama complejo de la gestión de las basuras urbanas e industriales en España, e intentar, de nuevo, paliar algunos de las graves deficiencias sectoriales y territoriales que hemos puesto de manifiesto.

\section{BIBLIOGRAFIA}

Alio, M. A. (1991): «Residus industrials a Catalunya». Revista Catalana de Geografía, n. ${ }^{\circ} 15$, vol. VI, 113-120

— y BRU, J. (1988): «Localización y control de los residuos sólidos industriales en Cataluña». Estudios Territoriales, n. $^{\circ} 28,143$ y ss.

ARCE, R.; Domínguez, R.; Menniti, S. y otros (1996): El medio ambiente en España. Madrid, Escuela de Organización Industrial-Mundi Prensa, $223 \mathrm{pp}$.

AYES, R. V. y KNEESE, A. V. (1973): «La polución y la calidad del medio ambiente urbano». En PERLOFF, H. S. (Ed.): La calidad del medio ambiente urbano. Barcelona, Oikos-Tau, pp. 43-73. 
FUNDACIÓN MAPFRE (1994): Estudio del estado del sector medioambiental en España. Madrid, Ed. Mapfre, $110 \mathrm{pp}$.

Martín, E. F. (1994): «Arma de doble filo. Pros y contras de los plásticos». Revista del $M O P T$, n. $^{\circ} 421,77-81$.

MARTín-CoBos, M. et al. (1983): «Deficiencias del servicio de recogida y tratamiento de basuras en el medio urbano andaluz». Revista de Estudios Regionales, n. ${ }^{\circ}$ 12, 291310.

ORDÓÑEZ, J. L. (1994): «Cada cosa en su sitio. Recogida selectiva de papel, cartón y vidrio». Revista del MOPT, n. $^{\circ} 420,33-37$.

Otero DEL PERAL, L. R. (1988): Residuos sólidos urbanos. Madrid, M.O.P.U., 78 pp.

O.C.D.E. (1997): Análisis de los resultados medioambientales. España. París, O.C.D.E., $211 \mathrm{pp}$.

RodRíguez, M. y Ruiz, G. (1983): «El ámbito local de una política de medio ambiente en Cataluña: El caso de los residuos sólidos». Revista de Estudios Regionales, n. ${ }^{\circ} 12$, 273-290.

TAPIA, F. y TOHaRIa, M. (1995): Medio Ambiente: ¿Alerta Verde? Madrid, Acento Editorial, $301 \mathrm{pp}$.

RESUMEN: Residuos sólidos urbanos e industriales: situación y gestión en España En el presente artículo se recogen diversos aspectos fundamentales para comprender la situación de los residuos sólidos urbanos e industriales y, específicamente, las causas de que éstos se hayan convertido en uno de los grandes retos de la política medioambiental española. Se analizan la producción y el tratamiento de los residuos, incidiendo en su evolución reciente, en los logros obtenidos y en las carencias estructurales que aún persisten. Éstas son el resultado de una gestión desigual e insuficiente, caracterizada, entre otras cosas, por la aparición de profundos contrastes territoriales que se destacan en el artículo. Finalmente se aborda el debate en torno al futuro de los residuos españoles y se analizan los grandes instrumentos planificadores que se han diseñado para hacer frente al problema.

PALABRAS ClAVE: Medio ambiente. Residuos sólidos urbanos. Residuos industriales. Política medioambiental.

ABSTRACT: Urban and Industrial Solid Waste: it's conditions and management in Spain. This article comes across some fundamental aspects to understand the situation of urban and industrial solid waste and, specifically, the different causes that explain the reason for become one of the main challenges of the spanish environmental politics. I analize the production and processing of waste, falling upon its recent evolution, the obtained achievements and some structural deficiencies that still remain, as a result of an unequal and insufficient management, characterized, among others, by the appearance of the deep territorial contrasts pointed in this article. Finally, I undertake the debate on the future of waste in Spain, studying the main planning instruments designed to face this problem.

KEY WORDS: Environment. Urban solid waste. Industrial waste. Environmental politics.

RESUMÉE: Résidus Solides Urbains e Industriels: situation et gestion en Espagne. Dans cet article on étudie divers aspects fondamentaux pour comprendre la situation des 
résidus solides urbains et industriels, et spécifiquement, les causes qui ont fait que ceux-ci soient devenus l'un des plus grands défis de la politique de l'environnement en Espagne. On analyse la production et le traitement des résidus, leur récente évolution, les réussites et les carences structurelles qui persistent encore. Ces dernières sont le résultat d'une gestion inégale et insuffisante caractérisée, entre autres choses, par le surgissement de profonds contrastes territoriaux soulignés dans l'article. Finalement on aborde le débat sur le futur des résidus espagnols et l'on analyse les grands instruments de planification dessinés pour affronter le problème.

Most CLÉS: Environnement. Résidus solides urbains. Résidus industriels. Politique de l'environnement. 